\title{
AVALIAÇÃO DE GENÓTIPOS TETRAPLOIDES DE BANANEIRA CULTIVADOS EM ÁREA INFESTADA PELO AGENTE CAUSAL DO MAL-DO-PANAMÁ ${ }^{1}$
}

\author{
SEBASTIÃO DE OLIVEIRA E SILVA² ${ }^{2}$ ARISTÓTELES PIRES DE MATOS ${ }^{2}$, \\ ZILTON JOSÉ MACIEL CORDEIRO², MATEUS JONNEI CARNEIRO LIMA ${ }^{3}$, \\ EDSON PERITO AMORIM ${ }^{2}$
}

RESUMO - O mal-do-Panamá é uma doença que causa grandes prejuízos à bananicultura no Brasil, uma vez que as principais cultivares em uso são suscetíveis ao Fusarium. Este trabalho teve como objetivos avaliar características agronômicas e resistência ao mal-do-Panamá de híbridos tetraploides de bananeira. O experimento foi conduzido no campo experimental da Embrapa Mandioca e Fruticultura Tropical, em delineamento casualizado, com 14 tratamentos e 10 repetições, nos anos de 2000 e 2001. Foram avaliados 14 tetraploides (FHIA-03, PV03-44, PC42-01, PV42-53, PV42-68, PV42-81, PV42-85, PV42-129, PV42-142, PV42-143, SH3640, ST12-31, ST42-08 e YB42-21) e a cultivar Maçã, usada como testemunha. Avaliaramse as características altura da planta $(\mathrm{m})$ e diâmetro do pseudocaule $(\mathrm{cm})$ a $30 \mathrm{~cm}$ do solo, peso da massa do cacho $(\mathrm{kg})$, penca $(\mathrm{kg})$ e fruto $(\mathrm{g})$, número de pencas por cacho, de frutos por penca e de dias do florescimento à colheita, e a incidência do mal-do-Panamá. As médias dos genótipos foram agrupadas pelo teste de Scott e Knott, a 5\% de probabilidade. Observa-se uma ampla variação genética nos caracteres avaliados. Os genótipos FHIA 03, ST12-31, SH3640, PV42-142, PV42-53 e PV42-68 apresentam boas características agronômicas e resistência ao mal-do-Panamá.

Termos para indexação: Musa spp., avaliação, melhoramento, Fusarium.

\section{EVALUATION OF TETRAPLOID GENOTYPES OF BANANA TREE CULTIVATED IN FUSARIUM-INFESTED AREA}

\begin{abstract}
Fusarium wilt is one of the major diseases which cause great damage to the banana crop in Brazil since most cultivars used are susceptible. This study was carried out to evaluate agronomical characteristics and resistance to Fusarium wilt in tetraploid banana hybrids. The experiment was led in the experimental field at Embrapa Cassava and Tropical Fruits in a complete randomized design with 14 treatments and 10 replicates in the years 2000 and 2001. Fourteen tetraploids were evaluated: FHIA-03, PV03-44, PC42-01, PV42-53, PV42-68, PV42-81, PV42-85, PV42-129, PV42-142, PV42-143, SH3640, ST12-31, ST42-08 and YB42-21 and the Silk cultivar used as the control. The following characteristics were evaluated: plant height $(\mathrm{m})$ and pseudostem diameter $(\mathrm{cm})$ at $30 \mathrm{~cm}$ from the ground, bunch weight $(\mathrm{kg})$, hand weight $(\mathrm{kg})$ and fruit weight $(\mathrm{g})$, number of hands per bunch, fruits per hand and days to flowering until harvest and Fusarium wilt incidence. The mean values of the genotypes were grouped by the Scott Knott test at 5\% significance. There is a broad variation regarding the characteristics evaluated. FHIA 03, ST12-31, SH3640, PV42-142, PV42-53 and PV42-68 present good agronomical characteristics as well as resistance to Fusarium wilt.
\end{abstract}

Index terms: Musa spp., evaluation, plant breeding, Fusarium.

${ }^{1}$ (Trabalho 033-10). Recebido em: 22-01-2010. Aceito para publicação em: 06-04-2010.

${ }^{2}$ Pesquisador(a) A, Dr(a). Embrapa Mandioca e Fruticultura Tropical, Rua Embrapa, s/nº - Cruz das Almas-BA, 44380-000. E-mail: ssilva@cnpmf.embrapa.br, zilton@cnpmf.embrapa.br, apmatos@cnpmf.embrapa.br, edson@cnpmf.embrapa.br.

${ }^{3}$ Graduando em Agronomia, Universidade Federal do Recôncavo da Bahia, Cruz das Almas-BA, E-mail: mateusjonnei@yahoo.com.br 


\section{INTRODUÇÃO}

A bananicultura possui grande importância econômica e social, sendo cultivada numa extensa área mundial, onde o Brasil é o quarto produtor, com uma produção de aproximadamente 6,9 milhões de toneladas em 2007, em uma área de 509 mil hectares (FAO, 2009).

A maioria das cultivares de bananeira utilizada pelos agricultores é suscetível às principais pragas da cultura, fato que conduz a severas perdas no rendimento, que podem alcançar 100\%, uma vez que as alternativas de controle apresentam-se pouco eficientes e/ou de custo elevado.

Uma das estratégias para a solução dos problemas mencionados é a criação de novas variedades produtivas e resistentes a pragas, por meio do melhoramento genético, que possibilita a obtenção de híbridos tetraploides e triploides superiores, mediante cruzamento de cultivares com diploides melhorados (SILVA et al., 1998; SILVA et al., 2002a).

O mal-do-Panamá é uma doença causada pelo fungo Fusarium oxysporum f.sp. cubense (Foc), considerada uma das mais importantes doenças da bananeira. Ele pode causar elevadas perdas na produção quando são utilizadas cultivares suscetíveis ao patógeno. A doença foi constatada pela primeira vez em 1874, em plantios de banana na Austrália. No Brasil, o primeiro relato da sua incidência foi confirmado no Estado de São Paulo, em 1930, na cultivar Maçã (KIMATI; GALLI, 1980). A doença atinge quase todos os locais onde se cultiva banana no País, sendo a Maçã a cultivar de maior suscetibilidade. O controle dessa doença é fundamentado no uso de medidas integradas, por meio da aplicação de práticas culturais e da utilização de variedades resistentes (CORDEIRO et al., 1993; SILVA et al., 2002a).

Este trabalho teve como objetivo avaliar características agronômicas e a reação ao Fusarium oxysporum f.sp. cubense de híbridos tetraploides melhorados de bananeira, cultivados em área artificialmente infestada com o patógeno.

\section{MATERIAL E MÉTODOS}

O trabalho foi realizado na Embrapa Mandioca e Fruticultura Tropical, em Cruz das Almas (BA), localizada a $12^{\circ} 40^{\prime} 39$ 'de latitude sul, $39^{\circ} 40^{\prime} 23^{\prime \prime}$ de longitude oeste, altitude de $220 \mathrm{~m}$, temperatura média de $24,5^{\circ} \mathrm{C}$, umidade relativa de $82 \%$, precipitação média anual de 1.197 mm (EMBRAPA, 1993). As avaliações foram realizadas nos anos de 2000 e 2001.
O local de avaliação foi artificialmente infestado com Fusarium oxysporum f.sp. cubense (Foc) mediante cultivo sucessivo de banana 'Maçã', altamente suscetível ao patógeno (CORDEIRO, et al., 1993). A fim de promover uma distribuição uniforme do inóculo, a área experimental foi arada e gradeada antes da implantação do experimento. No momento do plantio, uma muda da cultivar Maçã foi plantada ao lado de cada planta do genótipo a ser avaliado.

Foram avaliados 14 tetraploides (FHIA-03, PV03-44, PV42-01, PV42-53, PV42-68, PV4281, PV42-85, PV42-129, PV42-142, PV42-143, SH3640, ST12-31, ST42-08 e YB42-21), e a cultivar Maçã (Tabela 1) usada como testemunha, em delineamento experimental inteiramente casualizado, com 14 tratamentos e 10 repetições. Cada planta foi considerada uma repetição.

Os tratos culturais foram realizados de acordo com as recomendações técnicas (ALVES; OLIVEIRA, 1999) e o espaçamento utilizado foi de $3 \mathrm{mx}$ $2 \mathrm{~m}$ com irrigação. Por ocasião do florescimento do primeiro e segundo ciclos, foram avaliadas as características altura da planta $(\mathrm{m})$ e diâmetro do pseudocaule $(\mathrm{cm})$ a $30 \mathrm{~cm}$ do solo. Na colheita dos dois ciclos, avaliaram-se: peso da massa do cacho $(\mathrm{kg})$, penca $(\mathrm{kg})$ e fruto $(\mathrm{g})$, número de pencas por cacho, de frutos por penca e de dias do florescimento à colheita, além da resistência ao Fusarium.

A incidência do mal-do-Panamá foi avaliada com base na expressão dos sintomas internos da doença, mediante cortes transversais do rizoma e exame da descoloração vascular causada pela infecção por Foc, atribuindo-se notas conforme a escala proposta por Cordeiro et al. (1993), como segue: (0), ausência de descoloração vascular, planta sadia; (1), pontos isolados de descoloração no câmbio vascular; (2), descoloração correspondente a até $1 / 3$ do câmbio vascular; (3), descoloração entre 1/3 e 2/3 do câmbio vascular; (4), descoloração superior a 2/3 do câmbio vascular, e (5), descoloração total do câmbio vascular. A cultivar Maçã foi utilizada como padrão de suscetibilidade ao Foc e como testemunha para avaliação da doença.

Os resultados obtidos da caracterização agronômica e da incidência do mal-do-Panamá foram submetidos à análise da variância, e as médias, agrupadas pelo teste de Scott e Knott (1974), a 5\% de significância. Para análise de variância, os dados da avaliação do mal-do-Panamá foram transformados para $\sqrt{\mathbf{x}+0,5}$. 


\section{RESULTADOS E DISCUSSÃO}

Para todas as características avaliadas nos dois ciclos de produção, foi observada a formação de agrupamentos por meio do teste de Scott e Knott (1974) (Tabelas 2 e 3) . Como era de se esperar, as plantas da cultivar Maçã não chegaram a produzir, sendo, portanto, avaliadas somente para incidência da doença.

As médias de altura de planta no primeiro e segundo ciclos variaram de 2,34 $\mathrm{m}$ a 3,29 $\mathrm{m}$ e de $3.05 \mathrm{~m}$ a 4,21 m, respectivamente, sendo o genótipo SH3640 o mais baixo e o ST12-31 com maior altura de planta. Formaram-se dois agrupamentos no primeiro ciclo e quatro grupos de genótipos no segundo ciclo. Embora os números de grupos tenham sido diferentes, entre os dois ciclos, praticamente não houve mudança na ordem dos genótipos. Assim, os híbridos mais altos ou mais baixos comportaram-se como tal, nos dois ciclos. Como observado por outros autores, as plantas do segundo ciclo foram mais altas que as do primeiro (DONATO et al., 2006; LEDO et al., 2008). De acordo com Soto Ballestero (1992), a altura definitiva só é atingida a partir do terceiro ciclo, independentemente do híbrido; além disso, esse descritor apresenta grande importância sob o ponto de vista fitotécnico e de melhoramento genético, pois influi nos aspectos de densidade de plantio e manejo da cultura, interferindo na produção (BELALCÁZAR-CARVAJAL, 1991). A identificação de tetraploides melhorados com baixa estatura é importante, uma vez que a resistência à doença, associada ao porte baixo e boas características organolépticas dos frutos, pode trazer maior retorno econômico para o agricultor (LEITE et al., 2003).

Com relação ao diâmetro do pseudocaule, 0 menor valor observado no primeiro ciclo foi de 17,70 cm do genótipo ST42-08 e o maior foi de $24,33 \mathrm{~cm}$ do genótipo FHIA 03 (Tabela 2), no segundo ciclo, a variação foi de 22,12 cm do genótipo PV42-129 a $28,56 \mathrm{~cm}$ do híbrido FHIA 03 (Tabela 3). Por meio do teste de Scott e Knott (1974), foi observada a formação de três agrupamentos para essa característica, tanto no primeiro quanto no segundo ciclo. O diâmetro de pseudocaule é importante no melhoramento genético de bananeira, estando relacionado ao vigor, à resistência à quebra do pseudocaule refletindo na capacidade de sustentação do cacho. Assim, genótipos que apresentam maior diâmetro do pseudocaule são menos suscetíveis ao tombamento (SILVA, et al., 1999; SILVA et al., 2002a; DONATO, 2003).

Quanto à média do número de dias da emissão da inflorescência à colheita, a variação observada foi de 107 dias (SH3640) a 168 dias (PV42-81) no primeiro ciclo, e de 108,56 dias (SH3640) a 153,88 dias (PV42-85) no segundo ciclo, com a formação de dois grupos em ambos os ciclos (Tabelas 2 e 3 ). Grupamentos e valores semelhantes para essa característica têm sido observados por vários autores (SILVA et al., 2002b; DONATO et al., 2003; LEDO et al., 2008).

A variação para peso do cacho foi de 7,39 kg (PV03-44) a 27,78 kg (FHIA-03) considerando o primeiro e segundo ciclos (Tabelas 2 e 3). Plantas do segundo ciclo produziram cachos maiores, à exceção do YB42-21, que produziu um cacho menor, provavelmente, devido à presença do maldo-Panamá. Embora o valor elevado do coeficiente de variação (próximo a 27 \%), houve a formação de três grupos de genótipos no primeiro ciclo e de quatro agrupamentos no segundo ciclo. De forma geral, os genótipos que foram mais produtivos no primeiro ciclo também o foram no segundo. O peso do cacho expressa a capacidade produtiva do genótipo. No entanto, não pode ser considerado isoladamente na escolha de uma variedade, pois outros caracteres, também influenciam no processo de seleção, a exemplo, a qualidade do fruto: peso, comprimento, diâmetro, sabor e resistência ao despencamento e à doenças (SILVA et al., 2002a).

Quanto ao número de pencas, observou-se a formação de quatro agrupamentos no primeiro e de apenas dois grupos no segundo ciclo (Tabelas 2 e 3). Com relação a essa característica, o destaque foi da FHIA03, que apresentou o maior número de pencas por cacho, com média de 7,44 e 7,62 pencas no primeiro e segundo ciclos, respectivamente. Os piores desempenhos foram observados nos genótipos PV03-44, no primeiro ciclo, e YB42-21 no segundo ciclo, com médias de 5,60 e 5,80 pencas, respectivamente. O número de pencas é um caráter de grande interesse para o produtor e de importância fundamental para o melhoramento genético da bananeira, uma vez que a penca se constitui na unidade comercial, além do que, um aumento no número de pencas pode acarretar em elevação no peso do cacho, caráter que expressa a produtividade do genótipo (SILVA et al., 2002b; SILVA et al., 2006).

Para o caráter peso das pencas, foram observados quatro grupos dos tetraploides tanto no primeiro ciclo quanto no segundo (Tabelas 2 e 3 ). As maiores e as menores médias de peso de pencas do primeiro e segundo ciclos foram, respectivamente, dos genótipos FHIA03 $(25,19 \mathrm{~kg}$ e $25,87 \mathrm{~kg})$ e PV03-44 (5,60 kg e 8,50 kg).

O híbrido PV03-44 apresentou o menor peso de fruto no primeiro e segundo ciclos, $85,53 \mathrm{~g}$ e 99,09 g, respectivamente, enquanto o maior peso foi do FHIA 03 (212,37 g) no primeiro e do PV42- 
85 (216,96 g) no segundo ciclo. O peso médio dos frutos é um caráter importante para os trabalhos de melhoramento, e é influenciado pelas condições ambientais, sendo que não pode ser considerado isoladamente, mas sim associado a outros componentes que refletem a qualidade dos frutos, como o comprimento e o diâmetro do fruto (SILVA et al., 2002b).

Os menores números de frutos por penca, no primeiro e segundo ciclos, foram, respectivamente, dos genótipos PV42-01 (11,94) e PV42-129 (12,52). Os maiores valores dessa característica, nos dois ciclos, foram observados no FHIA 03. O número de frutos por penca é fundamental na determinação do tamanho e do peso do cacho, revelando importância no melhoramento genético e está diretamente relacionado com o número de pencas e de frutos por cacho (SILVA et al.,1999).

O peso de penca está estreitamente relacionado a fatores como peso e número de frutos. Essa correlação positiva é maior ou menor, a depender do genótipo e do meio ambiente. Por sua vez, o peso do cacho está relacionado aos caracteres do fruto e também ao número e peso das pencas (LIMANETO et al., 2003).

O híbrido FHIA 03, do grupo genômico $\mathrm{AABB}$, que pode ser consumido cru, sendo mais adequado para consumo cozido, apresentou o maior peso de cacho, peso de penca, número de pencas, número de frutos por penca e diâmetro de pseudocaule no primeiro e segundo ciclos. Por outro lado, o PV03-44, um dos primeiros híbridos selecionados na Embrapa, apresentou as menores médias para peso de cachos, número de pencas, peso de pencas e peso médio de frutos no primeiro ciclo. Vale ressaltar, no entanto, que nesse experimento não foram avaliadas as cultivares, Prata Comum, Yangambi Número 2, Prata São Tomé e Pacovan, genitoras femininas dos híbridos (PV42-01), (YB42-21), (ST42-08 e ST1231) e (PV42-142, PV42-53, PV42-68, PV42-85, PV42-81, PV42-129, PV42-143, PV03-44), respectivamente, e que se assim o fosse, provavelmente, teriam produção inferior aos respectivos híbridos, como foi observado por Donato et al. (2006).

Os resultados da análise da variância das notas atribuídas à descoloração vascular do rizoma na avaliação dos sintomas internos do mal-doPanamá, obtidos após a colheita do primeiro e segundo cachos, são mostrados nas Tabelas 2 e 3. A aplicação do teste de Scott e Knott (SKOTT; KNOTT, 1974), ao nível de 5\%, levou à formação de cinco grupos de genótipos no primeiro ciclo e quatro agrupamentos no segundo ciclo. Considera-se que, com uma incidência da doença inferior a 1,4, o genótipo é considerado resistente (CORDEIRO et al., 1993). O grupo e, do primeiro ciclo e os agrupamentos c e e, do segundo ciclo são constituídos de genótipos resistentes ao mal-do-Panamá. Dessa forma, classificaram como resistentes: PV42-53, PV42-68, PV42-81, PV42-142, PV42-143, ST42-08, ST12-31, PV03-44, FHIA-03 e SH36-40, no primeiro ciclo, e PV42-53, PV42-68, PV42-81, PV 42-85, PV42-142, PV42-143, ST42-08, ST12-31, PV03-44, FHIA-03 e SH36-40, no segundo ciclo. A reação de resistência ao mal-do-Panamá, constatada no híbrido tetraploide PV03-44, está de acordo com resultados já obtidos (CORDEIRO et al., 1993; MATOS et al., 1998). Por outro lado, PV42-129 e PC42-01, embora pertencendo a grupo diferente da variedade Maçã, padrão de suscetibilidade ao mal-do-Panamá, devem ser considerados suscetíveis a essa doença, tendo em vista que os mesmos mostraram infecções elevadas no primeiro e no segundo ciclos (nota $\geq 2,9$ ).

Conforme se pode observar nas Tabelas 2 e 3 , a testemunha suscetível ao mal-do-Panamá, 'Maçã', apresentou níveis máximos de infecção, representados pela nota 5,00 , no primeiro e segundo ciclos. O híbrido YB42-21, com intensidade de sintomas internos, do mal-do-Panamá, similar à da 'Maçã', foi classificado no mesmo grupo dessa cultivar, portanto, suscetível à doença. No entanto, o híbrido não chegou a entrar em colapso e foi capaz de produzir cachos, fato que o diferencia da banana 'Maçã', cujas plantas morreram antes da colheita, muitas delas antes mesmo da emissão do cacho.

Os resultados obtidos neste trabalho mostram ainda que sete dos dez híbridos que tiveram M53 (AA) como parental masculino (Tabela 1) apresentaram reação de resistência ao mal-do-Panamá, enquanto três expressaram graus variáveis de resistência, o que faz de M53 um parental promissor para utilização em programas de melhoramento genético da bananeira com o objetivo de gerar híbridos resistentes a $F$. oxysporum f.sp. cubense. A gradação nos níveis de resistência/tolerância ao mal-do-Panamá tem sido relatada em híbridos oriundos de cruzamentos envolvendo Calcutta 4 (AA) ou Lidi (AA) como parental masculino (CORDEIRO et al., 1993). 
TABELA 1 - Descrição dos genótipos de bananeira avaliados na Embrapa Mandioca e Fruticultura Tropical. Cruz das Almas-BA 2009.

Genótipo Grupo Genealogia Descrição do genótipo

FHIA-03 AABB SH3386 (ABB) x SH3320 (AA) Tipo figo, apresenta resistência à sigatokas amarela e negra.

ST12-31 AAAB Prata São Tomé (AAB) x Lidi (AA) Tipo Prata e resistente à sigatoka-amarela

SH3640 AAAB Prata-Anã (AAB) x SH3393 (AA) Tipo Prata e resistência parcial às sigatokas amarela e negra

PV42-142 AAAB Pacovan (AAB) x M53 (AA)

PV42-53 AAAB Pacovan (AAB) x M53 (AA)

PV42-68 AAAB Pacovan (AAB) x M53 (AA)

PV42-01 AAAB Prata Comum (AAB) x M53 (AA)

PV42-85 AAAB Pacovan (AAB) x M53 (AA)

PV42-81 AAAB Pacovan (AAB) x M53 (AA)

ST42-08 AAAB Prata São Tomé (AAB) x M53 (AA)

PV42-129 AAAB Pacovan (AAB) x M53 (AA)

YB42-21 AAAB Yangambi n 2 (AAB) x M53 (AA)

PV42-143 AAAB Pacovan (AAB) x M53 (AA)

PV03-44 AAAB Pacovan (AAB) x Calcutá (AA)

Maçã AAB Cultivar
Tipo Prata e resistente às sigatokas amarela e negra

Tipo Prata e resistente às sigatokas amarela e negra

Tipo Prata e resistente às sigatokas amarela e negra

Tipo Prata e resistente às sigatokas amarela e negra

Tipo Prata e resistente às sigatokas amarela e negra

Tipo Prata e resistente às sigatokas amarela e negra

Tipo Prata e resistente às sigatokas amarela e negra

Tipo Prata e resistente às sigatokas amarela e negra

Tipo Maçã e resistente à sigatoka-amarela

Tipo Prata resistente às sigatokas amarela e negra

Tipo Prata resistente às sigatokas amarela e negra

Maçã resistência parcial à sigatoka-amarela

TABELA 2- Médias de caracteres observados na época do florescimento e da colheita de 14 genótipos tetraploides de bananeira primeiro ciclo de produção. Cruz das Almas-BA 2009.

\begin{tabular}{lccccccccc}
\hline Genótipos & APL & DPC & NDE & PCA & NPE & PPE & PMF & NFP & AMP \\
\hline FHIA-03 & $2,61 \mathrm{~b}$ & $24,33 \mathrm{a}$ & $120,78 \mathrm{~b}$ & $27,64 \mathrm{a}$ & $7,44 \mathrm{a}$ & $25,19 \mathrm{a}$ & $212,37 \mathrm{a}$ & $15,72 \mathrm{a}$ & $0,20 \mathrm{e}$ \\
ST12-31 & $3,29 \mathrm{a}$ & $22,80 \mathrm{a}$ & $148,70 \mathrm{a}$ & $17,63 \mathrm{~b}$ & $6,50 \mathrm{~b}$ & $16,30 \mathrm{~b}$ & $172,15 \mathrm{~b}$ & $14,54 \mathrm{~b}$ & $0,00 \mathrm{e}$ \\
SH3640 & $2,34 \mathrm{~b}$ & $20,50 \mathrm{~b}$ & $107,00 \mathrm{c}$ & $15,34 \mathrm{~b}$ & $7,22 \mathrm{a}$ & $14,01 \mathrm{~b}$ & $151,47 \mathrm{~b}$ & $12,90 \mathrm{c}$ & $0,00 \mathrm{e}$ \\
PV42-142 & $3,07 \mathrm{a}$ & $19,58 \mathrm{~b}$ & $150,70 \mathrm{a}$ & $15,03 \mathrm{~b}$ & $6,30 \mathrm{~b}$ & $13,84 \mathrm{~b}$ & $160,33 \mathrm{~b}$ & $14,07 \mathrm{~b}$ & $0,00 \mathrm{e}$ \\
PV42-53 & $2,90 \mathrm{a}$ & $21,11 \mathrm{~b}$ & $154,33 \mathrm{a}$ & $14,86 \mathrm{~b}$ & $6,44 \mathrm{~b}$ & $13,86 \mathrm{~b}$ & $157,68 \mathrm{~b}$ & $13,70 \mathrm{~b}$ & $0,00 \mathrm{e}$ \\
PV42-68 & $3,03 \mathrm{a}$ & $20,63 \mathrm{~b}$ & $152,90 \mathrm{a}$ & $13,86 \mathrm{~b}$ & $6,50 \mathrm{~b}$ & $12,86 \mathrm{~b}$ & $155,00 \mathrm{~b}$ & $12,87 \mathrm{c}$ & $0,00 \mathrm{e}$ \\
PV42-01 & $2,89 \mathrm{a}$ & $20,43 \mathrm{~b}$ & $150,29 \mathrm{a}$ & $13,60 \mathrm{~b}$ & $5,85 \mathrm{c}$ & $12,47 \mathrm{~b}$ & $184,78 \mathrm{a}$ & $11,94 \mathrm{c}$ & $3,70 \mathrm{~b}$ \\
PV42-85 & $2,97 \mathrm{a}$ & $19,45 \mathrm{~b}$ & $156,40 \mathrm{a}$ & $13,24 \mathrm{~b}$ & $6,10 \mathrm{c}$ & $12,21 \mathrm{~b}$ & $154,72 \mathrm{~b}$ & $13,02 \mathrm{c}$ & $1,90 \mathrm{~d}$ \\
PV42-81 & $2,89 \mathrm{a}$ & $19,30 \mathrm{~b}$ & $168,00 \mathrm{a}$ & $12,80 \mathrm{~b}$ & $5,80 \mathrm{c}$ & $11,81 \mathrm{~b}$ & $162,34 \mathrm{~b}$ & $12,44 \mathrm{c}$ & $0,00 \mathrm{e}$ \\
ST42-08 & $2,98 \mathrm{a}$ & $17,70 \mathrm{c}$ & $154,90 \mathrm{a}$ & $11,90 \mathrm{c}$ & $5,70 \mathrm{c}$ & $10,98 \mathrm{c}$ & $157,78 \mathrm{~b}$ & $12,80 \mathrm{c}$ & $0,00 \mathrm{e}$ \\
PV42-129 & $2,86 \mathrm{a}$ & $18,05 \mathrm{c}$ & $135,50 \mathrm{~b}$ & $11,82 \mathrm{c}$ & $6,70 \mathrm{~b}$ & $10,89 \mathrm{c}$ & $129,19 \mathrm{c}$ & $12,61 \mathrm{c}$ & $2,90 \mathrm{c}$ \\
YB42-21 & $2,78 \mathrm{a}$ & $23,08 \mathrm{a}$ & $123,83 \mathrm{~b}$ & $11,40 \mathrm{c}$ & $4,67 \mathrm{c}$ & $10,56 \mathrm{c}$ & $141,73 \mathrm{~b}$ & $13,64 \mathrm{~b}$ & $4,56 \mathrm{a}$ \\
PV42-143 & $3,07 \mathrm{a}$ & $20,63 \mathrm{~b}$ & $141,00 \mathrm{a}$ & $9,53 \mathrm{c}$ & $6,00 \mathrm{c}$ & $8,34 \mathrm{~d}$ & $106,24 \mathrm{~d}$ & $13,18 \mathrm{c}$ & $0,00 \mathrm{e}$ \\
PV03-44 & $2,55 \mathrm{~b}$ & $18,05 \mathrm{c}$ & $150,80 \mathrm{a}$ & $7,39 \mathrm{c}$ & $5,60 \mathrm{~d}$ & $6,59 \mathrm{~d}$ & $85,53 \mathrm{~d}$ & $14,21 \mathrm{~b}$ & $0,50 \mathrm{e}$ \\
\hline Maçã & - & - & - & - & - & - & - & - & $5,00 \mathrm{a}$ \\
\hline CV\% & $\mathbf{9 , 2 5}$ & $\mathbf{1 0 , 7 0}$ & $\mathbf{1 0 , 6 9}$ & $\mathbf{2 6 , 6 7}$ & $\mathbf{1 4 , 1 9}$ & $\mathbf{2 2 , 1 8}$ & $\mathbf{2 6 , 7 9}$ & $\mathbf{1 3 , 7 1}$ & $\mathbf{2 3 , 0 5}$
\end{tabular}

APL: altura de planta (m); DPC: diâmetro do pseudocaule $(\mathrm{cm})$; NDE: número de dias da emissão à colheita; PCA: peso da massa do cacho $(\mathrm{kg})$; NPE: número de pencas; PPE: peso da massa das pencas $(\mathrm{kg})$; PMF: peso médio da massa dos frutos (g); NFP: número de frutos por penca; AMP: avaliação mal-do-Panamá. Médias seguidas pela mesma letra nas colunas não diferem estatisticamente entre si, pelo teste de Scott e Knott (1974), a $5 \%$ de probabilidade. Dados da avaliação do mal-do-Panamá foram transformados para $\sqrt{\mathrm{x}+0,5}$. 
TABELA 3 - Médias de caracteres observados na época do florescimento e da colheita de 14 genótipos tetraploides de bananeira no segundo ciclo de produção. Cruz das Almas-BA 2009.

\begin{tabular}{lccccccccc}
\hline Genótipos & APL & DPC & NDE & PCA & NPE & PPE & PMF & NFP & AMP \\
\hline FHIA-03 & $3,35 \mathrm{c}$ & $28,56 \mathrm{a}$ & $126,50 \mathrm{~b}$ & $27,78 \mathrm{a}$ & $7,62 \mathrm{a}$ & $25,87 \mathrm{a}$ & $209,27 \mathrm{a}$ & $16,06 \mathrm{a}$ & $1,10 \mathrm{c}$ \\
ST12-31 & $4,21 \mathrm{a}$ & $27,73 \mathrm{a}$ & $145,00 \mathrm{a}$ & $21,41 \mathrm{~b}$ & $7,57 \mathrm{a}$ & $19,90 \mathrm{~b}$ & $182,33 \mathrm{~b}$ & $14,53 \mathrm{~b}$ & $0,00 \mathrm{~d}$ \\
SH3640 & $3,05 \mathrm{~d}$ & $25,04 \mathrm{~b}$ & $108,56 \mathrm{~b}$ & $18,35 \mathrm{~b}$ & $7,67 \mathrm{a}$ & $17,72 \mathrm{~b}$ & $160,59 \mathrm{~b}$ & $13,54 \mathrm{~b}$ & $0,40 \mathrm{~d}$ \\
PV42-142 & $4,07 \mathrm{a}$ & $26,39 \mathrm{~b}$ & $152,11 \mathrm{a}$ & $19,10 \mathrm{~b}$ & $7,00 \mathrm{a}$ & $17,65 \mathrm{~b}$ & $186,56 \mathrm{a}$ & $13,55 \mathrm{~b}$ & $0,00 \mathrm{~d}$ \\
PV42-53 & $3,75 \mathrm{~b}$ & $27,05 \mathrm{a}$ & $135,00 \mathrm{~b}$ & $19,56 \mathrm{~b}$ & $7,70 \mathrm{a}$ & $18,32 \mathrm{~b}$ & $168,82 \mathrm{~b}$ & $13,95 \mathrm{~b}$ & $0,00 \mathrm{~d}$ \\
PV42-68 & $4,12 \mathrm{a}$ & $24,66 \mathrm{~b}$ & $146,25 \mathrm{a}$ & $19,16 \mathrm{~b}$ & $6,87 \mathrm{a}$ & $17,78 \mathrm{~b}$ & $195,08 \mathrm{a}$ & $13,25 \mathrm{~b}$ & $0,00 \mathrm{~d}$ \\
PV42-01 & $3,95 \mathrm{~b}$ & $22,56 \mathrm{c}$ & $126,56 \mathrm{~b}$ & $13,75 \mathrm{c}$ & $6,22 \mathrm{~b}$ & $12,93 \mathrm{c}$ & $158,55 \mathrm{~b}$ & $12,89 \mathrm{~b}$ & $3,70 \mathrm{~b}$ \\
PV42-85 & $4,07 \mathrm{a}$ & $25,13 \mathrm{~b}$ & $153,88 \mathrm{a}$ & $19,35 \mathrm{~b}$ & $6,75 \mathrm{~b}$ & $18,16 \mathrm{~b}$ & $216,96 \mathrm{a}$ & $13,29 \mathrm{~b}$ & $1,10 \mathrm{c}$ \\
PV42-81 & $3,99 \mathrm{~b}$ & $26,03 \mathrm{~b}$ & $138,13 \mathrm{a}$ & $16,77 \mathrm{~b}$ & $6,37 \mathrm{~b}$ & $15,64 \mathrm{~b}$ & $179,55 \mathrm{~b}$ & $13,92 \mathrm{~b}$ & $0,20 \mathrm{~d}$ \\
ST42-08 & $3,85 \mathrm{~b}$ & $25,77 \mathrm{~b}$ & $148,22 \mathrm{~b}$ & $15,15 \mathrm{c}$ & $6,55 \mathrm{~b}$ & $14,05 \mathrm{c}$ & $157,59 \mathrm{~b}$ & $13,71 \mathrm{~b}$ & $0,00 \mathrm{~d}$ \\
PV42-129 & $3,36 \mathrm{c}$ & $22,12 \mathrm{c}$ & $121,38 \mathrm{~b}$ & $11,39 \mathrm{~d}$ & $7,12 \mathrm{a}$ & $9,98 \mathrm{~d}$ & $113,18 \mathrm{c}$ & $12,52 \mathrm{~b}$ & $3,40 \mathrm{~b}$ \\
YB42-21 & $3,19 \mathrm{~d}$ & $25,14 \mathrm{~b}$ & $130,00 \mathrm{~b}$ & $9,64 \mathrm{~d}$ & $5,80 \mathrm{~b}$ & $8,89 \mathrm{~d}$ & $131,59 \mathrm{c}$ & $13,05 \mathrm{~b}$ & $4,56 \mathrm{a}$ \\
PV42-143 & $4,19 \mathrm{a}$ & $24,72 \mathrm{~b}$ & $133,11 \mathrm{~b}$ & $14,05 \mathrm{c}$ & $6,00 \mathrm{~b}$ & $13,00 \mathrm{c}$ & $160,20 \mathrm{~b}$ & $13,41 \mathrm{~b}$ & $0,00 \mathrm{~d}$ \\
PV03-44 & $3,56 \mathrm{c}$ & $22,70 \mathrm{c}$ & $124,70 \mathrm{~b}$ & $9,78 \mathrm{~d}$ & $6,40 \mathrm{~b}$ & $8,75 \mathrm{~d}$ & $99,09 \mathrm{c}$ & $13,90 \mathrm{~b}$ & $0,00 \mathrm{~d}$ \\
\hline Maçã & - & - & - & - & - & - & - & - & $5,00 \mathrm{a}$ \\
\hline CV\% & $\mathbf{9 , 5 0}$ & $\mathbf{1 1 , 3 0}$ & $\mathbf{1 2 , 0 0}$ & $\mathbf{2 6 , 8 0}$ & $\mathbf{1 5 , 0 0}$ & $\mathbf{2 0 , 0 0}$ & $\mathbf{2 8 , 0 0}$ & $\mathbf{1 5 , 4 0}$ & $\mathbf{2 3 , 3 5}$ \\
\hline
\end{tabular}

APL: altura de planta (m); DPC: diâmetro do pseudocaule (cm); NDE: número de dias da emissão à colheita; PCA: peso da massa do cacho (kg); NPE: número de pencas; PPE: peso da massa das pencas (kg); PMF: peso médio da massa dos frutos (g); NFP: número de frutos por penca; AMP: avaliação mal-do-Panamá. Médias seguidas pela mesma letra nas colunas não diferem estatisticamente entre si, pelo teste de Scott e Knott (1974), a 5 \% de probabilidade. Dados da avaliação do mal-do-Panamá foram transformados para $\sqrt{x+0,5} \cdot$ A tabela apresenta os dados originais.

\section{CONCLUSÕES}

1-Há ampla variação genética para todos os caracteres agronômicos nos genótipos de bananeira avaliados.

2-Os melhores genótipos são: o Tipo Figo FHIA 03 e os Tipo Prata ST12-31, SH3640, PV42142, PV42-53 e PV42-68, porque apresentam boas características agronômicas e resistência ao maldo-Panamá.

\section{REFERÊNCIAS}

ALVES, E.J.; OLIVEIRA, M.A. Práticas culturais. In: ALVES, E.J. (Org.). A cultura da Banana: aspectos técnicos, socieconômicos e agroindustriais. 2. ed. Brasília: Embrapa-SPI/ Embrapa-CNPMF, 1999. p.335-352.

BELALCÁZAR-CARVAJAL, S.L. El cultivo del plátano em el trópico. Cali: Imprensora Feriva, 1991. 376p. (IICA, Manual de assistência técnica, $50)$.
CORDEIRO, Z.J.M.; SHEPHERD, K.; SOARES FILHO, W.S; DANTAS, J.L.L. Avaliação de resistência ao mal-do-Panamá em híbridos tetraploides de bananeira. Fitopatologia Brasileira, Brasília, v.18, n.4, p.478-483, 1993.

DONATO, S.L.R. Comportamento de variedades e híbridos de bananeira (Musa spp.), em primeiro ciclo de produção no sudoeste da Bahia, região de Guanambi. 2003. 115 f. Dissertação (Mestrado em Ciência e Tecnologia de Sementes) - Faculdade de Agronomia "Eliseu Maciel”, Universidade Federal de Pelotas, Pelotas. 2003.

DONATO, S.L.R.; SILVA, S.O.; LUCCA FILHO, O.A.; LIMA, M.B.; DOMINGUES, H.; ALVES, J.S. Comportamento de variedades e híbridos de bananeira (Musa spp.), em dois ciclos de produção no sudoeste da Bahia. Revista Brasileira de Fruticultura, Jaboticabal, v.28, n.1, p.139-144, 2006. 
EMBRAPA. Serviço Nacional de Levantamento e Conservação de Solos. Levantamento detalhado dos solos do Centro Nacional de Pesquisa de Mandioca e Fruticultura Tropical. Cruz das Almas: Embrapa-CNPMF, 1993. 126p. (Boletim de Pesquisa, 7).

FAO. Food and Agriculture Organization of the United Nations. Acesso em: 27 maio 2009. Disponível em: <www.faostat.fao.org/site/340/default.aspx>.

KIMATI, H.; GALLI, F. Doenças da bananeira Musa spp., In: GALLI, F. Manual de fitopatologia: doenças das plantas cultivadas. São Paulo: Ceres, 1980. p.87-110.

LEDO, A. S.; SILVA JÚNIOR, J. F.; LEDO, C.A.S; SILVA, S.O. Avaliação de genótipos de bananeira na região do Baixo São Francisco, Sergipe. Revista Brasileira de Fruticultura, Jaboticabal, v. 30, n. 3, p. 691-695, 2008.

LEITE, J.B.V.; SILVA, S.O.; ALVES, E.J.; LINS, R.D.; JESUS, O.N. Caracteres da planta e do cacho de genótipos de bananeira, em quatro ciclos de produção, em Belmonte, Bahia. Revista Brasileira de Fruticultura, Jaboticabal, v.25, n.3, p.443- 447, 2003.

LIMA NETO, F. P.; SILVA, S.O.; FLORES, J. C. O. ; JESUS, O. N.; PAIVA, L. E. Relações entre caracteres de rendimento e de desenvolvimento em genótipos de bananeira. Magistra, Cruz das Almas, v. 15 , n. 2 , p. $275-281,2003$.

MATOS, A.P. de; BORGES, M. de F.; SILVA, S. de O. e; CORDEIRO, Z.J.M.; ANDRADE, S. de M. Reaction of banana genotypes to Fusarium wilt (Fusarium oxysporum f.sp. cubense) under field conditions in Brazil. In: Reunion ACORBAT, 13., 1998. Guayaquil, Ecuador. Memorias... p. 311-318.
SCOTT, A.J.; KNOTT, M.A. A cluster analysis method for grouping means in the analysis of variance. Biometrics, Washington, v.30, n.3, p.507-512, 1974.

SILVA, E. A.; BOLIANI, A.C.; CORRÊA, L.S. Avaliação de cultivares de bananeira (Musa sp.) na região de Selvíria-MS. Revista Brasileira de Fruticultura, Jaboticabal, v.28, n.1, p.101-103, 2006.

SILVA, S. O.; ALVES, E.J.; LIMA, M.B.; SILVEIRA, J.R.S. Bananeira. In: BRUCKNER, C.H. (Org.). Melhoramento de fruteiras tropicais. Viçosa-MG, 2002a. p.101-157.

SILVA, S. O.; ALVES, E.J.; SHEPHERD, K.; DANTAS, J.L.L. Cultivares, In: ALVES, E.J. (Org.). A cultura da banana: aspectos técnicos, socieconômicos e agroindustriais. 2. ed. Brasília: Embrapa-SPI/ Embrapa-CNPMF, 1999. p.85-105.

SILVA, S. O.; FLORES, J. C. O.; LIMA NETO, F. P. Avaliação de cultivares e híbridos de bananeira em quatro ciclos de produção. Pesquisa Agropecuária Brasileira, Brasília, v. 37, n. 11, p. 1567-1574, 2002b.

SILVA, S.O.; MATOS, A.P.; ALVES, E.J. Melhoramento genético da bananeira. Pesquisa Agropecuária Brasileira, Brasília, v.33, n.5, p.693-703, 1998.

SOTO BALLESTERO, M. Banana: cultivo e Comercialización. San José. Litografia y Imprensa, 1992. p. 170-204. 